\title{
Autoeficacia académica y su relación con el rendimiento académico en estudiantes de nutrición
}

\author{
Academic Self-Efficacy and Its Relationship With Academic Performance in Nutrition Students
}

\section{Auto-eficácia acadêmica e sua relação com o desempenho acadêmico em estudantes de nutrição}

\author{
Recibido • Received • Recebido: 21 / 11 / 2018 \\ Corregido • Revised • Revisado: 07 / 04 / 2020 \\ Aceptado • Accepted • Aprovado: 07 / 07 / 2020
}

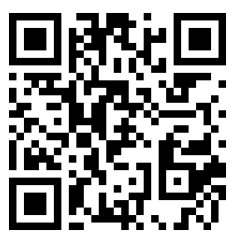

Cynthia Anahi Rosales-Ronquillo Universidad Juárez del Estado de Durango

Durango, México

cynthya_ronquillo@hotmail.com

https://orcid.org/0000-0001-5573-363X

Luis Fernando Hernández-Jácquez Universidad Pedagógica de Durango

Durango, México

Ifhj1@hotmail.com

https://orcid.org/0000-0002-6017-3267

\begin{abstract}
Resumen: El presente artículo muestra los resultados de una investigación que tuvo como objetivos principales determinar el nivel de autoeficacia académica percibida, e identificar la relación existente entre dicha autoeficacia y el rendimiento académico en la totalidad del estudiantado (391) de la Licenciatura en Nutrición de una facultad en el Estado de Durango, México. Su fundamento es la teoría de la autoeficacia de Albert Bandura, se desarrolló un estudio cuantitativo, no experimental y transversal; se utilizó la encuesta como técnica de recolección de información, y se obtuvo como resultados la percepción de parte del estudiantado de un nivel alto de autoeficacia académica y una relación positiva entre ésta y su rendimiento académico. A nivel general, se recomienda la intervención en aspectos como la concentración, la atención, y la confianza para intercambiar puntos de vista con el docente o la docente; aspectos todos que resultaron con bajas autovaloraciones.
\end{abstract}

Palabras claves: Autoeficacia académica; evaluación de aprendizaje; educación superior; nutrición.

Abstract: This paper shows the results of a research study whose main objectives were to determine the level of perceived academic self-efficacy and identify the relationship between self-efficacy and academic performance in all students (391) of the Nutrition Degree program of a faculty in the state of Durango, Mexico. Taking Albert Bandura's theory of self-efficacy as a theoretical basis, a quantitative, 
http://doi.org/10.15359/ree.24-3.7

http://www.una.ac.cr/educare

educare@una.cr

non-experimental, and transversal research study. A survey was applied, and the results obtained showed that the students present a high level of academic self-efficacy and that there is a positive relationship between this self-efficacy and the students' academic performance. It is recommended to intervene in aspects such as concentration, attention, and confidence to exchange points of view with the teacher; all these aspects showed low self-evaluations.

Keywords: Academic self-efficacy; learning assessment; higher education; nutrition.

Resumo: Este documento mostra os resultados de uma pesquisa cujos objetivos principais foram determinar o nível de auto eficácia acadêmica percebida e identificar a relação entre auto eficácia e desempenho acadêmico em todos os estudantes (391) do Curso de Nutrição de um faculdade no estado de Durango, México. Tomando como base teórica a Teoria da Auto Eficiência de Albert Bandura, foi desenvolvido um estudo quantitativo, não-experimental e transversal, utilizando a pesquisa como técnica de recuperação de informações, que mostrou que os estudantes possuem um alto nível de auto eficácia acadêmica, e que há uma relação positiva entre isso e seu desempenho acadêmico. Recomenda-se a intervenção em aspectos como concentração, atenção e confiança para trocar pontos de vista com o professor; todos os aspectos, o que resultou em baixas autoavaliações.

Palavras-chave: auto eficácia acadêmica; avaliação de aprendizagem; educação superior; nutrição.

\section{Introducción}

En años recientes el estudio de la autoeficacia ha comenzado a tomar fuerza en lo que a investigaciones reportadas en el idioma español se refiere, y en lo particular circunscrita al ámbito educativo se le ha utilizado como elemento predictor de conductas y situaciones académicas, tal como fue objeto de la presente investigación.

Para dar muestra de lo anterior y dentro del contexto mexicano, se tiene el estudio realizado por Hernández Jácquez (2018), quien determinó el perfil de autoeficacia académica percibida asociado a diversas variables sociales, demográficas y académicas en 182 estudiantes de educación superior, variables entre las cuales se encuentra el promedio de calificaciones obtenido en el semestre inmediato anterior al que se cursa. En este estudio se precisó que el estudiantado contaba con un nivel medio de autoeficacia académica.

El mismo Hernández Jácquez en el año 2015 llevó a cabo un estudio correlacional, transversal y no experimental con estudiantes de educación superior tecnológica con la intención de verificar la relación entre la autoeficacia académica y el estrés en 117 estudiantes, resultando que los sujetos estudiados presentaron un nivel de autoeficacia académica percibida medio y un nivel alto de estrés académico; con relación entre ambos elementos.

En este mismo año, Manzanera Jáuregui y Barraza Macías (2015) realizaron un estudio correlacional, no experimental y transversal, para determinar las expectativas de autoeficacia en estudiantado normalista. El resultado más sobresaliente fue el hecho de que el estudiantado contaba con un alto nivel de autoeficacia académica, más alto en las mujeres que en los varones.

Cynthia Anahi Rosales-Ronquillo y Luis Fernando Hernández-Jácquez

Los artículos de la Revista Electrónica Educare del Centro de Investigación y Docencia en Educación de la Universidad Nacional, Costa Rica, se comparten bajo términos de la Licencia Creative Commons: Reconocimiento, No Comercial, Sin Obra Derivada 3.0 Costa Rica. Las autorizaciones adicionales a las aquí delimitadas se pueden obtener en el correo: educare@una.cr 
http://doi.org/10.15359/ree.24-3.7

Con una muestra de 282 sujetos con edades entre 20 y 21 años de edad, Aguirre et al. (2015) analizaron la autoeficacia percibida en estudiantado universitario mexicano. Siguiendo un enfoque cuantitativo con un diseño descriptivo, los resultados revelaron que "las mujeres en comparación con los hombres reportan puntuaciones más altas en autoeficacia percibida y con mayor necesidad y posibilidad de ser más autoeficaces" (p. 97), lo cual sugiere, entonces, que a nivel docente "al diseñar cualquier tipo de intervención que tenga como objetivo la mejora de la autoeficacia percibida, habrá que tomar en cuenta a la variable género" (Aguirre et al., 2015, p. 97).

En el año 2014, Alpuche Hernández y Vega Pérez utilizaron la autoeficacia como insumo para predecir el comportamiento lector a través de un estudio no experimental correlacional, cuyo objetivo fue "identificar si la autoeficacia en la lectura predice el comportamiento lector en estudiantes de primer grado de primaria" (Alpuche Hernández y Vega Pérez, 2014, p. 241). En la investigación participaron 350 niños a quienes se les administraron dos instrumentos, y se concluyó que "a partir de las creencias de autoeficacia en la lectura, el estudiantado incorpora en su repertorio acciones y estrategias necesarias para desarrollar con efectividad su comportamiento lector" (Alpuche Hernández y Vega Pérez, 2014, p. 241).

Con las variables autoeficacia académica y estrés, en el año 2013, Hernández Jácquez y Barraza Macías realizaron un estudio correlacional, transversal y no experimental, para determinar la posible relación entre la autoeficacia académica percibida y el estrés en 68 estudiantes de posgrado relacionados con temáticas educativas. Encontraron que las personas encuestadas mostraron un nivel de autoeficacia percibida alto; sin embargo, solamente una de las dimensiones del constructo presentó una relación negativa con el estrés.

Por su parte, Galicia-Moyeda et al. (2013) realizaron un estudio sobre la autoeficacia en escolares adolescentes y su relación con ciertas variables, entre las cuales se encontraba el rendimiento académico. Participaron 80 estudiantes de educación secundaria en quienes se encontró una relación positiva entre ambas variables. En el mismo año, Pool-Cibrián y MartínezGuerrero (2013) investigaron sobre la relación entre la autoeficacia, las metas de aprendizaje y los problemas de concentración en una muestra de 766 estudiantes de una universidad pública de México e identificaron asociaciones entre la autoeficacia percibida con metas de aprendizaje.

Por último BlancoVega et al. (2012) muestrearon un total de 2089 estudiantes de universidad para determinar el perfil de autoeficacia académica percibida en la conducta académica entre hombres y mujeres. El resultado reveló que tomando como base el perfil de autoeficacia, las mujeres mostraron un mayor deseo de éxito en sus estudios escolares que los hombres.

Si bien, en general las investigaciones han mostrado que existe una relación positiva entre la autoeficacia académica y aspectos académicos, no fue posible localizar resultados que lo avalen en estudiantes de licenciaturas asociadas a la salud (en el contexto mexicano) con características muy particulares, tales como la responsabilidad que deben asumir ante un 
http://doi.org/10.15359/ree.24-3.7

http://www.una.ac.cr/educare

educare@una.cr

diagnóstico o valoración de pacientes; el manejo de emociones, sentimientos y actitudes, y la prácticamente infalible intervención que deben llevar a cabo; situaciones que se van modulando a través de su proceso académico.

De manera particular en la práctica diaria de una determinada Facultad del Estado de Durango, México, el estudiantado de la Licenciatura en Nutrición está entrando en una dinámica en la que cada día muestra menor interés por aprender, tiene menor confianza en sí mismo y no le entusiasma el aprender cosas nuevas de la profesión que eligió ejercer, situación que comienza a repercutir en su rendimiento académico. En el día a día es fácil percatarse de esta condición (según algunas personas docentes) ya que el estudiantado suele faltar a clases y al realizar actividades como debates, trabajo en equipos o exposiciones, muestra inseguridad aun cuando conoce el tema. Al momento de encomendarles una tarea, gran cantidad de estudiantes solo copian y pegan la información que localizan en internet, en sus evaluaciones se conforman con la calificación mínima aprobatoria y en general no cuentan con los conocimientos necesarios en cada una de las materias que cursan. Cabe resaltar que también hay estudiantado interesado en aprender y obtener las mejores calificaciones, pero lamentablemente la cultura del menor esfuerzo parece estar permeando las aulas de la Facultad.

Algunas de estas situaciones pudiesen asociarse con la percepción que el estudiantado tiene de sí mismo y baja autoestima e inseguridad, pero sobre todo con su falta de confianza para llevar a bien sus actividades académicas tanto presentes como futuras, lo que puede impactar, a su vez, en su rendimiento académico.

Así, considerando lo anterior y el vacío encontrado en la revisión de antecedentes, se plantearon los siguientes los objetivos de investigación:

1. Identificar el nivel de autoeficacia académica percibida en el estudiantado de la Licenciatura en Nutrición de la Facultad.

2. Determinar la relación entre autoeficacia académica percibida y el rendimiento académico en el estudiantado de la Licenciatura en Nutrición de la Facultad.

3. Determinar la relación entre ciertas variables sociales y la autoeficacia académica percibida en el estudiantado de la Licenciatura en Nutrición de la Facultad.

\section{Marco teórico}

Finalizando la década de los setenta es postulada la teoría de la autoeficacia como "producto principalmente de dos ideas que Bandura tiene en mente, la primera es que las intervenciones que modifican el ambiente son eficaces para modificar la conducta y la segunda que la intervención cognitiva del sujeto es imprescindible"Valencia (2006, citado por Velásquez Fernández, 2012, p. 150).

4 
http://doi.org/10.15359/ree.24-3.7

Así, el concepto de autoeficacia surgió dentro de la teoría cognitiva social (Bandura, 1999) y dentro de sus aportaciones más importantes se destaca la consideración del funcionamiento de las personas como una interacción tríadica entre la conducta, los factores cognitivos y los sucesos ambientales.

Bandura (1995) concibe la autoeficacia como los juicios de las personas acerca de sus capacidades para organizar y ejecutar acciones necesarias para manejar situaciones futuras. Además, el mismo Bandura (1997) y Pajares (2002) indican que las personas autoeficaces poseen un autosistema que les permite ejercer un control sobre el medio ambiente por encima de sus pensamientos, sus sentimientos y sus acciones.

Bardales, Díaz, Jiménez, Terreros y Valencia (2006, citados por Velásquez, 2012, p. 150) la definen como el "estado psicológico en el cual la persona evalúa su capacidad y habilidad de ejecutar determinada tarea, actividad y conducta en una situación específica con un nivel de dificultad previsto". Además, Valiante (2000) señala a la autoeficacia como un importante mediador cognitivo de competencia y rendimiento.

Así pues, Pajares (1996) ha dejado entrever, en repetidas ocasiones, la fuerza de la autoeficacia como una variable capaz de predecir el logro académico. Este constructo psicológico también se ha definido como la capacidad percibida de hacer frente a situaciones específicas, que involucran la creencia acerca de las propias capacidades para organizar y ejecutar acciones, para alcanzar determinados resultados (Bandura, 1986).

De manera general, Bandura (1997) propone cuatro fuentes de información que causan que las percepciones de autoeficacia se desarrollen y cambien:

a) Experiencias personales o de dominio: Son la fuente de información más importante de autoeficacia y básicamente se refiere a que "el éxito repetido en determinadas tareas aumenta las evaluaciones positivas de autoeficacia, mientras que los fracasos repetidos las disminuyen, especialmente cuando los fracasos no pueden atribuirse a un esfuerzo insuficiente o a circunstancias externas" (Olaz, 2003, Cuestiones conceptuales acerca de la autoeficacia, párr. 4).

b) Experiencias vicarias: Describe la importancia de observar que si otras personas ejecutan exitosamente ciertas actividades, el sujeto puede llegar a creer que él mismo posee las capacidades suficientes para desempeñarse con el mismo éxito.

c) Persuasión verbal: Se trata de convencer al sujeto receptor de que es capaz de lograr sus objetivos siempre y cuando sea dedicado y se esfuerce lo suficiente, sin embargo, si fracasa, el ente receptor perderá la confianza en la persona que lo persuade, por eso "es más fácil disminuir la autoeficacia por medio de la persuasión que incrementarla" (Bandura, 1997, citado por Tuckman y Monetti, 2011, p. 396). 
http://doi.org/10.15359/ree.24-3.7

http://www.una.ac.cr/educare

educare@una.cr

d) Activación fisiológica: En esta fuente se destaca cómo las personas que presentan nerviosismo, temor y ansiedad son más susceptibles a estresarse y así reducir su nivel de autoeficacia percibida. Si se reducen estos factores, aumenta el nivel de autoeficacia y, por consecuencia, el desempeño mejora (Tuckman y Monetti, 2011).

Por su parte, la variable rendimiento académico es multidimensional y multifactorial, y como lo señala Lamas (2015, p. 315):

La complejidad del rendimiento académico se inicia desde su conceptualización. En ocasiones se le denomina aptitud escolar, desempeño académico o rendimiento escolar, pero, generalmente, las diferencias de concepto solo se explican por cuestiones semánticas ya que se utilizan como sinónimos.

En este espectro multifactorial se pueden mencionar aspectos internos y externos que configuran ese rendimiento, tales como los propios del estudiantado: motivación intrínseca, capacidades cognitivas, habilidades de procesamiento de la información, movilización de saberes, relaciones familiares, etcétera; pero también se encuentran los aspectos relacionados con el sistema educativo, como el estilo de enseñanza empleado por el personal docente, los materiales, el acceso a tecnologías, las prácticas de campo o de laboratorio, el propio currículo y hasta la disciplina, entre otros.

Así pues, es evidente que el rendimiento académico debiese ser el reflejo de todo lo anterior, según Nieto Martín (2008, p. 255) lo explica:

El rendimiento académico no sóloes utilizado por los profesionales dela educación, sinoque llega a esgrimirse como bandera de la calidad o no de un determinado sistema educativo... Sin embargo, las variables que operan en el hecho de educarse son demasiado numerosas como para que, en su mayor parte, escapen del control de los propios educadores; aun así, el rendimiento educativo es la razón de ser de las instituciones escolares: y éste es un dato crucial para conocer, comprender e interpretar la vida en el aula y cada uno de los elementos personales que la constituyen.

Es entonces cuando, para fines de este estudio, se tomó como base lo expuesto por Pérez, Ramón y Sánchez (2000), Vélez Van y Roa (2005) citados por Garbanzo (2007, p. 46), quienes lo conceptualizan así:

La suma de diferentes y complejos factores que actúan en la persona que aprende, ... y que ha sido definido con un valor atribuido al logro del estudiante en las tareas académicas. Se mide mediante las calificaciones obtenidas con una valoración cuantitativa cuyos resultados muestran las materias ganadas o perdidas, la deserción y el grado de éxito académico. 
http://doi.org/10.15359/ree.24-3.7

En este sentido, Cascón (2000, citado por Navarro, 2003, p. 4) atribuye la importancia de utilizar las calificaciones como mejor elemento para aproximarse al rendimiento académico por dos situaciones:

1) los problemas sociales, y no sólo académicos, que están ocupando a los entes responsables políticos, profesionales de la educación, padres y madres de estudiantes; y a la ciudadanía en general, son la consecución de un sistema educativo efectivo y eficaz que proporcione al alumnado el marco idóneo donde desarrollar sus potencialidades; 2) el indicador del nivel educativo adquirido... en ... la ... totalidad de los países desarrollados y en vías de desarrollo, han sido, siguen y probablemente seguirán siendo las calificaciones escolares. A su vez, éstas son reflejo de las evaluaciones o exámenes donde el alumnado ha de demostrar sus conocimientos sobre las distintas áreas o materias que el sistema considera necesarias y suficientes para su desarrollo como miembro activo o activa de la sociedad.

\section{Metodología}

La presente investigación se desarrolló desde el enfoque cuantitativo, de alcance correlacional y transeccional, dado que se pretende identificar la relación entre las variables ya descritas sin pretender atribuir relaciones de causa-efecto (Salkind, 1999) en un momento único de tiempo; y de tipo no experimental, ya que "no se posee control directo [sobre] las variables... debido a que sus manifestaciones ya han ocurrido..." (Kerlinger y Lee, 2002, p. 504).

Fundamentado en los antecedentes y en el planteamiento del problema, se establecieron dos hipótesis de investigación:

$\mathrm{H}_{1}$ : El nivel de autoeficacia académica del estudiantado de la Licenciatura en Nutrición de la Facultad, es medio.

$\mathrm{H}_{2}$ : Existe una relación positiva entre el nivel de autoeficacia académica y el rendimiento académico en el estudiantado de la Licenciatura en Nutrición de la Facultad.

Como técnica para la recuperación de información se recurrió a la encuesta, y a nivel instrumental se utilizó “El inventario de expectativas de autoeficacia académica" (IEAA) (Barraza Macías, 2010), para conocer el nivel de autoeficacia del estudiantado. Asociado al rendimiento académico, se consideró el promedio de bachillerato para el estudiantado de primer semestre y para el inscrito del segundo al octavo semestre, el promedio del semestre inmediato anterior.

Respecto al IEAA, se compone de 20 ítems que pueden ser contestados en un escalamiento tipo Likert de cuatro valores: nada seguro, poco seguro, seguro y muy seguro. De acuerdo con Barraza Macías (2010), los ítems se distribuyen en tres dimensiones: actividades académicas de insumo para el aprendizaje, actividades académicas orientadas a la producción, y actividades 
http://doi.org/10.15359/ree.24-3.7

http://www.una.ac.cr/educare

educare@una.cr

académicas de interacción para el aprendizaje. El instrumento, reportó una confiablidad de .91 en alfa de Cronbach y de .88 en la confiabilidad por mitades, además reportó validez de consistencia interna, pues todos los ítems mostraron una correlación positiva (con un nivel de significación de .00) con el puntaje global obtenido por cada sujeto encuestado. En cuanto al análisis de grupos contrastados resultó adecuado (todos los ítems permiten discriminar, con un nivel de significación de .00) entre los grupos que reportan un alto y bajo nivel de expectativas de autoeficacia académica. La estructura tridimensional del instrumento explica el $49 \%$ de la varianza total, obtenida mediante análisis factorial (Barraza Macías, 2010).

Al inventario descrito anteriormente se agregó un pequeño apartado con información social y académica de interés para el estudio.

Las personas participantes de esta investigación fueron los 391 estudiantes de la Licenciatura en Nutrición de la Facultad (por lo tanto, el estudio fue censal): 288 fueron mujeres y 103 hombres, quienes, en el momento de la recolección de datos, cursaban desde el primero al octavo semestre.

La edad del estudiantado osciló entre los 18 y los 33 años. Del total de las personas encuestadas, el 23.3 \% (91 estudiantes) se ubicó en los 21 años de edad mientras que las demás personas estuvieron entre los 29 y los 33 años (1 estudiante por cada año de edad). La mayor cantidad del estudiantado se encontraba en la edad regular para estudiantes de licenciatura (18 a 23 años).

Para tener certeza de la forma de distribución de los datos y, por consiguiente, del tipo de pruebas estadísticas a emplear, se utilizó la prueba normalidad de Kolmogorov-Smirnov, la que resultó con una significación de .514, lo cual indica que los datos siguen el modelo de una distribución normal y, por lo tanto, las pruebas a utilizar corresponden a estadística paramétrica.

Así pues, para dar cuenta de la estadística descriptiva de ambas variables, se utilizó la media aritmética (promedio) y la desviación estándar como medida de tendencia central y de variabilidad, respectivamente. Respecto a la estadística inferencial, para calcular la asociación o relación entre el rendimiento académico y la autoeficacia académica se calculó el coeficiente de correlación de Pearson, y para los análisis de diferencia de grupo de la autoeficacia académica respecto al género y al semestre de estudio, se empleó la prueba t de Student para muestras independientes y el análisis de varianza de un factor, respectivamente, en ambas pruebas se consideró un valor de significación de 0.050 .

\section{Resultados, análisis y discusión}

El promedio de bachillerato fue recabado solo para el estudiantado de primer semestre (46 sujetos), osciló del 6.8 al 9.8 y el más representativo fue el 8.0 con $17.4 \%$ del estudiantado, seguido de 8.50 y 8.80 , cada uno con el $8.7 \%$ del estudiantado. 
http://doi.org/10.15359/ree.24-3.7

Si se considera una clasificación de bajo, medio y alto en cuanto al promedio, en donde de 6.0 a 7.3 sea un nivel bajo, de 7.4 a 8.7 un nivel medio, y de 8.8 a 10.0 un nivel alto, por tanto, el estudiantado del primer semestre tiene un nivel medio de rendimiento académico ya que su promedio general fue de 8.27 .

Del segundo semestre en adelante se recabó el promedio del semestre inmediato anterior, el cual varió de 6.0 a 10.0, lo que mostró una gran dispersión en los resultados. El promedio más frecuente se situó en el rango de 8.01 a 8.50 con un porcentaje de $31.0 \%$, seguido de 7.51 a 8.00 con $21.7 \%$ y 8.51 a 9.00 con $20.1 \%$ del estudiantado.

Tomando en cuenta la misma clasificación que se estableció para el promedio de bachillerato, se puede plantear que el estudiantado del segundo al octavo semestre presenta un nivel medio de rendimiento académico, pues su promedio general fue de 8.49.

En otro sentido, como se explicó anteriormente, la variable autoeficacia académica se compone de tres dimensiones que analizadas de manera descriptiva arrojaron los datos que se muestran en la Tabla 1.

Tabla 1: Estadística descriptiva para la autoeficacia académica (y sus dimensiones)

\begin{tabular}{lcc}
\hline \multicolumn{1}{c}{ Dimensiones de autoeficacia } & Media aritmética & Desviación estándar \\
\hline Actividades académicas de insumo para el aprendizaje & 2.08 & .4381 \\
Actividades académicas orientadas a la producción & 2.16 & .4478 \\
Actividades académicas de interacción para el aprendizaje & 1.98 & .5137 \\
Autoeficacia general & 2.07 & .4153 \\
\hline
\end{tabular}

Nota: Elaboración propia.

De acuerdo con Barraza Macías (2010), para interpretar los puntajes de autoeficacia es necesario transformarlos en porcentaje a través de una regla de tres simple, en donde un nivel bajo corresponde de 0 a 33\%, un nivel medio de 34 a $66 \%$ y un nivel alto de 67 a 100\%.

De esta manera, las actividades académicas de insumo para el aprendizaje equivalen a $69.33 \%$, es decir, un nivel alto. Dentro de estas, las mayormente valoradas resultaron ser la seguridad para"buscar la información necesaria para elaborar un ensayo o un artículo académico sin importar si es en una biblioteca o en la internet" (Barraza Macías, 2010, p. 10), y el "tomar notas de los aspectos más importantes que se abordan durante las clases" (Barraza Macías, 2010, p. 10); lo cual evidencia que la actividad académica de sistematización de información está dominada por el estudiantado. Por otro lado, la confianza para concentrarse a la hora de estudiar sin distraerse y el prestarle atención a la clase que imparte el docente o la docente, 
http://doi.org/10.15359/ree.24-3.7

http://www.una.ac.cr/educare

educare@una.cr

resultó con la menor puntuación, lo que puede indicar que hay problemas relacionados con la atención dada durante el trabajo individual (al estudiar) y al recibir la cátedra el profesorado.

Las actividades académicas orientadas a la producción equivalen al $72 \%$, lo que representa un nivel alto; destaca la valoración positiva en cuanto a la confianza para realizar cualquier trabajo académico que le encargue el docente o la docente y la comprensión de la idea central de un texto o los aspectos medulares de la exposición del personal docente o compañero o compañera; mientras que los asuntos menos autovalorados fueron la confianza para construir argumentos propios en los trabajos escritos y aprobar cualquier proceso de evaluación, sin importar el docente o la docente o el seminario.

De lo anterior, llama la atención como el estudiantado refiere confianza al realizar cualquier producto académico; sin embargo, no se siente capaz de argumentar con sus propios fundamentos lo que ahí se plasma. Esto podría explicar por qué la elaboración de aquellos productos que impliquen habilidades de análisis, la reflexión o crítica, no les resulta tan fácil; mientras aquellos que requieren habilidades de sistematización, síntesis o el resumir pueden desarrollarse de mejor manera.

Las actividades académicas de interacción correspondieron al 66\%, un nivel medio. Dentro de éstas, las más altas puntuaciones se obtuvieron en lo que corresponde a la seguridad de poder realizar una buena exposición de alguno de los contenidos del seminario y trabajar eficazmente en cualquier equipo, sin importar quiénes sean los compañeros o compañeras que lo integren; mientras que las más bajas puntuaciones resultaron en la seguridad para poder cuestionar al docente o la docente cuando no se está de acuerdo en lo que se expone, así como en la confianza para preguntarle cuando no se entiende algo de lo que se está abordando.

De lo anterior, se puede afirmar que el estudiantado se siente capaz de brindar una buena explicación de alguna temática, tanto por la preparación que realiza del contenido como por sus habilidades para transmitir esa temática. También, se siente seguro de colaborar con cualquiera de sus pares en las actividades en equipo de trabajo. Por lo contrario, cualquier actividad relacionada con poder intercambiar o cuestionar puntos de vista del personal docente muestra una confianza baja, lo que se puede interpretar como inseguridad para sostener una conversación académica fundamentada con alguien a quien se le suele considerar con más información (preparación); aspecto que coincide con la baja autovaloración que se obtuvo también para poder construir argumentos propios en los trabajos escritos de la dimensión anterior.

De manera global, la media aritmética de autoeficacia académica percibida equivale a $69 \%$, un valor que se sitúa en el límite inferior del nivel alto.

En otro orden de ideas, para analizar las correlaciones de la autoeficacia respecto al promedio de bachillerato y al promedio del semestre anterior (ver Tabla 2), se utilizó el coeficiente de correlación de Pearson. 
http://doi.org/10.15359/ree.24-3.7

http://www.una.ac.cr/educare educare@una.cr

Tabla 2: Correlación de Pearson de autoeficacia con promedio de bachillerato y promedio del semestre Anterior

\begin{tabular}{lcc}
\hline \multicolumn{1}{c}{ Dimensiones de autoeficacia } & $\begin{array}{c}\text { Promedio de } \\
\text { bachillerato }\end{array}$ & $\begin{array}{c}\text { Promedio del semestre } \\
\text { anterior }\end{array}$ \\
\hline Actividades académicas de insumo para el aprendizaje & .068 & $.159^{*}$ \\
Actividades académicas orientadas a la producción & .107 & $.232^{*}$ \\
Actividades académicas de interacción para el aprendizaje & .115 & $.199^{*}$ \\
Autoeficacia general & .117 & $.222^{*}$ \\
\hline
\end{tabular}

Nota: *Correlación significativa al nivel 0.01 (bilateral). Elaboración propia.

En cuanto al promedio de bachillerato (para estudiantes de primer semestre), no pudo determinarse una relación estadísticamente significativa con alguna de las dimensiones de autoeficacia, ni con el nivel general, lo que significa que el promedio que el estudiantado haya obtenido en su bachillerato, no incide sobre la percepción de autoeficacia académica. En esta situación, el resultado se aleja de lo explicado por Bandura (1977) respecto a las fuentes de autoeficacia y, sobre todo, de la experiencia directa, como mayor insumo para ella. Sin embargo, esto podría explicarse por el amplio contexto académico de donde proviene el estudiantado, dado que una parte ha cursado bachilleratos relacionados con las humanidades y ciencias sociales; otra, con relacionados con las ciencias exactas; otra más egresó de bachilleratos generales, e incluso, preparatorias relacionadas con actividades agropecuarias; por lo que, en realidad, nadie tiene una referencia directa en relación con las ciencias de salud y, por ende, no se tiene un referente previo de la dificultad (o facilidad) que esto representaría.

Contrario a lo sucedido con el promedio de bachillerato, el promedio del semestre anterior (para estudiantes de segundo a octavo semestre) y la autoeficacia académica, incluidas sus tres dimensiones, mostraron correlaciones significativas (al nivel de 0.01). Además, todas estas correlaciones resultaron positivas, lo que significa que conforme aumenta el promedio que el estudiantado haya obtenido en el semestre inmediato anterior, su nivel de autoeficacia, tanto de manera global como en las dimensiones, también aumenta. En caso contrario, al disminuir el promedio obtenido disminuye su autoeficacia percibida.

En este resultado se tiene una total concordancia con la fuente directa de autoeficacia que es, para Bandura (1977), la que provee la mayor fuerza de autovaloración. Evidentemente, una vez que el estudiantado ha transitado al segundo semestre y de éste a los posteriores, conoce las generalidades y pormenores de la licenciatura tanto en el plano académico (relacionado con la esencia misma de las ciencias de la salud) como escolar (relacionado con la institución), situación que tiene influencia en los logros (o fracasos) esperados dependiendo de los obtenidos.

En el sentido positivo, el haber obtenido un promedio bien valorado le permitirá, a cada estudiante, cierto control sobre sus pensamientos, sentimiento y acciones (Bandura, 1997; Pajares, 2002) que ayudará al éxito académico futuro. En el sentido negativo, un promedio que no es bien 
http://doi.org/10.15359/ree.24-3.7

http://www.una.ac.cr/educare

educare@una.cr

visto por el propio sujeto, puede hacer que pensamientos, sentimientos y acciones de desilusión o frustración influyan de manera determinante en el fracaso escolar; ello alude a la autoeficacia como variable psicológica determinante y predictora del logro académico (Pajares, 1996).

Respecto a las variables sociales de interés en el estudio, se tiene que para conocer si existen diferencias de género en la población en términos de autoeficacia académica, se empleó la prueba t de Student para muestras independientes con un nivel de prueba de 0.050 (ver Tabla 3).

Tabla 3: Autoeficacia académica en relación al género

\begin{tabular}{lccc}
\hline Dimensiones de autoeficacia & $\mathrm{t}$ & $\mathrm{gl}$ & Sig. (bilateral) \\
\hline Actividades académicas de insumo para el aprendizaje & .732 & 384 & .465 \\
Actividades académicas orientadas a la producción & .899 & 379 & .369 \\
Actividades académicas de interacción para el aprendizaje & 2.836 & 384 & .005 \\
Autoeficacia general & 1.762 & 387 & .079 \\
\hline
\end{tabular}

Nota: Elaboración propia.

Únicamente la dimensión de actividades académicas de interacción muestra una diferencia estadísticamente significativa, con una media aritmética mayor los hombres (2 106 puntos) que las mujeres (1 939 puntos).

En lo que respecta al semestre, el análisis se llevó a cabo mediante un ANOVA, resultando que únicamente no existe una diferencia estadísticamente significativa en la dimensión actividades académicas de insumo para el aprendizaje (ver Tabla 4). Para las demás dimensiones se procedió a realizar la prueba de intervalos de Duncan para determinar en qué semestres se encuentra la diferencia.

Tabla 4: Autoeficacia académica en relación al semestre de estudio

\begin{tabular}{lcc}
\hline \multicolumn{1}{c}{ Dimensiones de autoeficacia } & $\mathrm{F}$ & Sig. \\
\hline Actividades académicas de insumo para el aprendizaje & 1.863 & .074 \\
Actividades académicas orientadas a la producción & 4.422 & .000 \\
Actividades académicas de interacción para el aprendizaje & 2.922 & .005 \\
Autoeficacia general & 3.399 & .002 \\
\hline
\end{tabular}

Nota: Elaboración propia. 
http://doi.org/10.15359/ree.24-3.7

De acuerdo con la prueba de intervalos de Duncan, en la dimensión actividades académicas orientadas a la producción de información el nivel de autoeficacia más alto se obtuvo en el estudiantado de octavo semestre (2 403 puntos), mientras que el más bajo fue en el segundo semestre (1 997 puntos), situación un tanto normal, ya que en el estudiantado que ha llegado a los últimos semestres han pasado por una formación y una serie de actividades que les ha permitido "dominar" las actividades orientadas a la producción; escenario totalmente contrapuesto para quienes cursan los primeros

Para la dimensión actividades académicas de interacción, el nivel de autoeficacia más elevado se obtuvo en el estudiantado de octavo semestre ( 2226 puntos) a diferencia del estudiantado de tercer semestre, quienes muestran la menor autoeficacia (1 865 puntos), lo que indica que el estudiantado de octavo semestre han adoptado a lo largo de la carrera mejores estrategias para trabajar eficazmente con cualquier compañero, competir académicamente con cualquier compañero o compañera, hacer aportaciones a la clase, realizar una buena exposición, preguntar a su docente o cuestionarle cuando no estén de acuerdo con lo expuesto, mientras que el estudiantado de tercer semestre no cuenta con la misma seguridad ya que va comenzando y aprendiendo poco a poco a realizar estas actividades con mayor eficacia.

Por último, la autoeficacia general del estudiantado fue más alta en octavo semestre (2 295 puntos), a diferencia del segundo semestre, pues presentaron la menor autoeficacia (1 978 puntos), lo que nuevamente parecería una situación normal, ya que el estudiantado de los semestres inferiores proviene de bachillerato con orientaciones diversas, lo que les dificulta el adaptarse a los métodos, discursos, materiales y textos de las ciencias de la salud. Por el contrario, el estudiantado del último semestre parece ya dominar lo anterior.

\section{Conclusiones}

Partiendo de las acotaciones de Bandura (1997), quien considera la autoeficacia como los juicios de las personas acerca de sus capacidades para alcanzar determinados niveles de rendimiento, y del primer objetivo de investigación, identificar el nivel de autoeficacia académica percibida en el estudiantado, se concluye que dicho nivel fue alto, lo que significa que el estudiantado se siente capaz de realizar, en lo general, las actividades académicas que se llevan a cabo en la Facultad. Con esto, se rechaza la hipótesis de investigación que señalaba que este nivel de autoeficacia sería medio $\left(\mathrm{H}_{1}\right)$.

Llevando a cabo un análisis intravariable, tanto las actividades de insumo como las de producción para el aprendizaje resultaron con una valoración alta, pero las actividades de retroalimentación o de interacción solo alcanzaron el nivel medio, lo que evidencia un área de oportunidad de mejora, sobre todo en lo que respecta a la confianza para discutir, sostener y defender sus ideas de forma argumentada con el profesorado, al que normalmente se le 
http://doi.org/10.15359/ree.24-3.7

http://www.una.ac.cr/educare

educare@una.cr

considera un modelo. Esto pudiese tener dos aristas de análisis: la primera, la propia experiencia negativa del estudiantado al intercambiar puntos de vista con el profesorado, y la segunda, la observación de que sus pares tampoco han tenido éxito en ello. Esto es justamente a lo que Bandura (1977) se refiere con la experiencia vicaria que, sin lugar a dudas, influye en la percepción de fracaso al intentar realizar la actividad.

De manera general, el alto nivel de autoeficacia coincide con lo encontrado por Hernández Jácquez y Barraza Macías (2013) con el estudiantado de posgrado y con lo reportado con el estudiantado de una escuela normal en el año 2015 por Manzanera Jáuregui y Barraza Macías. Estos dos estudios se realizaron en el mismo Estado que el presente, por lo que se puede pensar que existe cierto perfil en cuanto a autoeficacia académica se refiere. Sin embargo, Hernández Jácquez $(2015,2018)$ señaló que el estudiantado de educación superior obtuvo un nivel medio de autoeficacia, por lo que la suposición del perfil queda para corroborarse en futuras investigaciones.

El segundo objetivo de la investigación fue determinar la relación entre autoeficacia académica percibida y el rendimiento académico en el estudiantado, relación que resultó positiva para el estudiantado del segundo semestre en adelante, mientras que para el del primer semestre no fue posible determinar asociación estadística significativa alguna. Esta discrepancia en el resultado puede explicarse, como ya se comentó, desde la perspectiva de los variados perfiles que ingresan al primer semestre y que no se relacionan de manera directa con la Licenciatura en Nutrición. Desde el segundo semestre, todo el estudiantado tiene la misma base a partir de la cual construye su percepción de autoeficacia. Con esto, se acepta la segunda hipótesis de investigación $\left(\mathrm{H}_{2}\right)$, con la salvedad ya comentada: existe una relación positiva entre el nivel de autoeficacia académica y el rendimiento académico en el estudiantado de la Licenciatura en Nutrición de la Facultad.

Esta relación es coincidente con lo encontrado por Hernández Jácquez (2018) en educación superior y con Galicia-Moyeda et al. (2013) en educación secundaria. Además son similares, en cuanto a la relación positiva entre la autoeficacia e indicadores académicos como las metas de aprendizaje (Pool-Cibrián y Martínez-Guerrero, 2013) y el comportamiento lector (Alpuche Hernández y Vega Pérez, 2014); es decir, se confirma lo señalado en los antecedentes: la autoeficacia es un buen predictor de situaciones académicas.

En relación con el tercer objetivo de investigación, determinar la relación entre ciertas variables sociales y la autoeficacia percibida, resulta que el semestre es una variable que hace diferencia entre los niveles de autoeficacia, el estudiantado del último semestre (octavo) es el que posee el mayor nivel mientras que el del segundo, la más baja autoeficacia. En cuanto al género, no se encontró diferencia alguna respecto a la autoeficacia general, más que únicamente con la dimensión de actividades académicas de interacción para el aprendizaje en donde los varones 
http://doi.org/10.15359/ree.24-3.7

resultaron con mayor nivel que las mujeres. Aunque sólo resultó en esta dimensión, llama la atención que el resultado es contrario a lo reportado en los antecedentes consultados, pues, en todos, las mujeres reportaron mayor nivel que los hombres (Aguirre et al., 2015; Blanco Vega et al., 2012; Manzanera Jáuregui y Barraza Macías, 2015; Blanco Vega et al., 2012).

Con los datos obtenidos, se recomienda la intervención en aquellos aspectos que resultaron con bajas autovaloraciones, porque, sin duda, son elementos imprescindibles en la confianza para desarrollarse de mejor manera como estudiante. Con especial hincapié resultaría importante trabajar actividades para la mejora de la concentración hacia el estudio y la atención selectiva en las clases, ambos elementos forman parte de las actividades de insumo para el aprendizaje y que, de atenderse, es muy probable que se mejoren las actividades encaminadas a la realización de productos académicos. También se sugiere trabajar la confianza para que el estudiantado pueda conversar o intercambiar puntos de vista con su docente, parte de las actividades de interacción para el aprendizaje, y que, de hacerse de manera seria, profesional y fundamentada, suele generar un enorme crecimiento en el estudiantado.

Por último, quedan como futuras líneas el investigar más al respecto de otras variables de índole psicológica que pudiesen estar relacionadas con la problemática estudiada, ya que, si bien la autoeficacia aporta en su comprensión, es solamente una perspectiva de las múltiples que tiene el fenómeno. En otro ámbito de investigación, la conformación de un perfil general de autoeficacia (si es que este existe) del estudiantado universitario en el Estado de Durango podría determinarse con los aportes de otros estudios que enriquezcan lo que se tiene hasta el momento.

\section{Declaración de Material complementario}

Este artículo tiene disponible como material complementario:

- La versión preprint del artículo en https://doi.org/10.5281/zenodo.3384266

\section{Referencias}

Aguirre, J. F., Blanco, J. R., Rodríguez-Villalobos, J. M. y Ornelas, M. (2015). Autoeficacia general percibida en universitarios mexicanos, diferencias entre hombres y mujeres. Formación Universitaria, 8(5), 97-102. http://dx.doi.org/10.4067/S0718-50062015000500011

Alpuche Hernández, A. y Vega Pérez, L. O. (2014). Predicción del comportamiento lector a partir de la autoeficacia. Revista Mexicana de Investigación Educativa, 19(60), 241-266. http:// www.scielo.org.mx/pdf/rmie/v19n60/v19n60a11.pdf 
http://doi.org/10.15359/ree.24-3.7

http://www.una.ac.cr/educare

educare@una.cr

Bandura, A. (1977). Self-efficacy: Toward a unifying theory of behavioral change. Psychological Review, 84(2), 191-215. http://dx.doi.org/10.1037/0033-295X.84.2.191

Bandura, A. (1986). Social fundations of thought and action. A social cognitive theory. Prentice Hall.

Bandura, A. (1995). Self-efficacy in changing societies. Cambidge University Press. https://doi. org/10.1017/CBO9780511527692

Bandura, A. (1997). Self-efficacy: The exercise of control. N. H. Freeman.

Bandura, A. (1999). Social cognitive theory: An agentic perspective. Asian Journal of Social Psychology, 2, 21-41. https://doi.org/10.1111/1467-839X.00024

Barraza Macías, A. (2010). Validación del inventario de expectativas de autoeficacia académica en tres muestras secuenciales e independientes. CPU-e, Revista de Investigación Educativa, 10, 1-30. https://doi.org/10.25009/cpue.v0i10.65

Blanco Vega, H., Ornelas Contreras, M., Aguirre Chávez, J. F. y Guedea Delgado, J. C. (2012). Autoeficacia percibida en conductas académicas. Diferencias entre hombres y mujeres. Revista Mexicana de Investigación Educativa, 17(53), 557-571. http://www.scielo.org.mx/ pdf/rmie/v17n53/v17n53a11.pdf

Galicia-Moyeda, I. X., Sánchez-Velasco, A. y Robles-Ojeda, F. J. (2013). Autoeficacia en escolares adolescentes: Su relación con la depresión, el rendimiento académico y las relaciones familiares. Anales de Psicología, 29(2), 491-500. https://doi.org/10.6018/ analesps.29.2.124691

Garbanzo Vargas, G. (2007). Factores asociados al rendimiento académico en estudiantes universitarios, una reflexión desde la calidad de la educación superior pública. Revista Educación, 31(1), 43-63. https://doi.org/10.15517/revedu.v31i1.1252

Hernández Jácquez, L. F. (2015). Autoeficacia y estrés académico en educación superior tecnológica. Cognition et Doctrina. Cognición y Aprendizaje en los Agentes Educativos, 1(1), 23-40. http://200.23.125.59/PDF/Cognicion/Revista/CognitionEtDoctrina1.pdf

Hernández Jácquez, L. F. (2018). Perfil sociodemográfico y académico en estudiantes universitarios respecto a su autoeficacia académica percibida. Psicogente, 21(39), 35-49. http://doi.org/10.17081/psico.21.39.2820

Hernández Jácquez, L. F. y Barraza Macías, A. (2013). Rendimiento académico y autoeficacia percibida. Un estudio de caso. Instituto Universitario Anglo Español. http://iunaes.mx/wpcontent/uploads/2014/08/FINAL-LIBRO-DE-AUTOEFICACIA-DE-LUIS-FERNANDO.pdf

Kerlinger, F. N. y Lee, H. B. (2002). Investigación del comportamiento. Métodos de investigación en ciencias sociales (4. a ed.). McGraw Hill. 
Lamas, H. A. (2015). Sobre el rendimiento escolar. Propósitos y Representaciones, 3(1), 313-386. http://dx.doi.org/10.20511/pyr2015.v3n1.74

Manzanera Jáuregui, M. E. y Barraza Macías, A. (2015). Expectativas de autoeficacia académica en alumnos normalistas del Estado de Durango. En L. F. Hernández Jácquez (Coord.), Autorregulación académica. Proceso desde la sociación de los estudiantes (pp. 153-182). Instituto Universitario Anglo Español. http://iunaes.mx/wp-content/uploads/2015/02/ Libro-Luis-Fdo.-Autorregulaci\%C3\%B3n-Acad\%C3\%A9mica.-Proceso-desde-laasociaci\%C3\%B3n-de-los-estudiantes.pdf

Navarro, R. E.(2003). El rendimiento académico: Concepto, investigación y desarrollo. REICE. Revista Iberoameticana sobre Calidad, Eficacia y Cambio en Educación, 1(2), 1-16. https:// revistas.uam.es/index.php/reice/issue/view/379

Nieto Martín, S. (2008). Hacia una teoría sobre el rendimiento académico en enseñanza primaria a partir la investigación empirica: Datos preliminares. Teoría de la Educación. Revista Interuniversitaria, 20, 249-274. https://revistas.usal.es/index.php/1130-3743/article/ view/992

Olaz, F. O. (2003). Autoeficacia, diferencias de género y comportamiento vocacional. Revista Electrónica de Motivación y Emoción, 6(13), 1-20. http://reme.uji.es/articulos/ aolazf5731104103/texto.html

Pajares, F. (1996). Self-efficacy beliefs in academic settings. Review of Educational Research, 66(4), 543-578. http://dx.doi.org/10.2307/1170653

Pajares, F. (2002). Self-efficacy beliefs in academia context:s An outline. https://www.uky. edu/ eushe2/Pajares/efftalk.html

Pool-Cibrián, W. J. y Martínez-Guerrero, J. I. (2013). Autoeficacia y uso de estrategias para el aprendizaje autorregulado en estudiantes universitarios. RevistaElectrónica de Investigación Educativa, 15(3), 21-36. http://redie.uabc.mx/vol15no3/contenido-pool-mtnez.html

Salkind, N. J. (1999). Métodos de investigación (3. a ed.). Prentice Hall.

Tuckman, B. W. y Monetti, D. M. (2011). Psicología educativa. Cengage Learning.

Valiante, G. (2000). Writing self-efficacy and gender orientation. A developmental perspective, a dissertation proposal. Emory University.

Velásquez Fernández, A. (2012). Revisión histórico-conceptual del concepto de autoeficacia. Revista Pequén, 2(1), 148-160. http://www.ubiobio.cl/miweb/webfile/media/265/8\%20 REVISI\%C3\%93N\%20HIST\%C3\%93RICO-CONCEPTUAL\%20DEL\%20CONCEPTO\%20 DE\%20AUTOEFICACIA.pdf 